\title{
Total IgA and IgA reactivity to antigen I/II epitopes in HLA-DRB1*04 positive subjects
}

\author{
V. Wallace McCarlie ${ }^{1^{*}}$, James K. Hartsfield Jr. ${ }^{2}$, Janice S. Blum ${ }^{3}$, Carlos González-Cabezas ${ }^{4}$, \\ Judith R. Chin ${ }^{5}$, George J. Eckert ${ }^{6}$, Lorri A. Morford ${ }^{7}$, Mark D. Pescovitz ${ }^{8 \#}$, Henry Rodriguez ${ }^{9}$, \\ Margherita Fontana ${ }^{4}$, Richard L. Gregory ${ }^{10^{*}}$

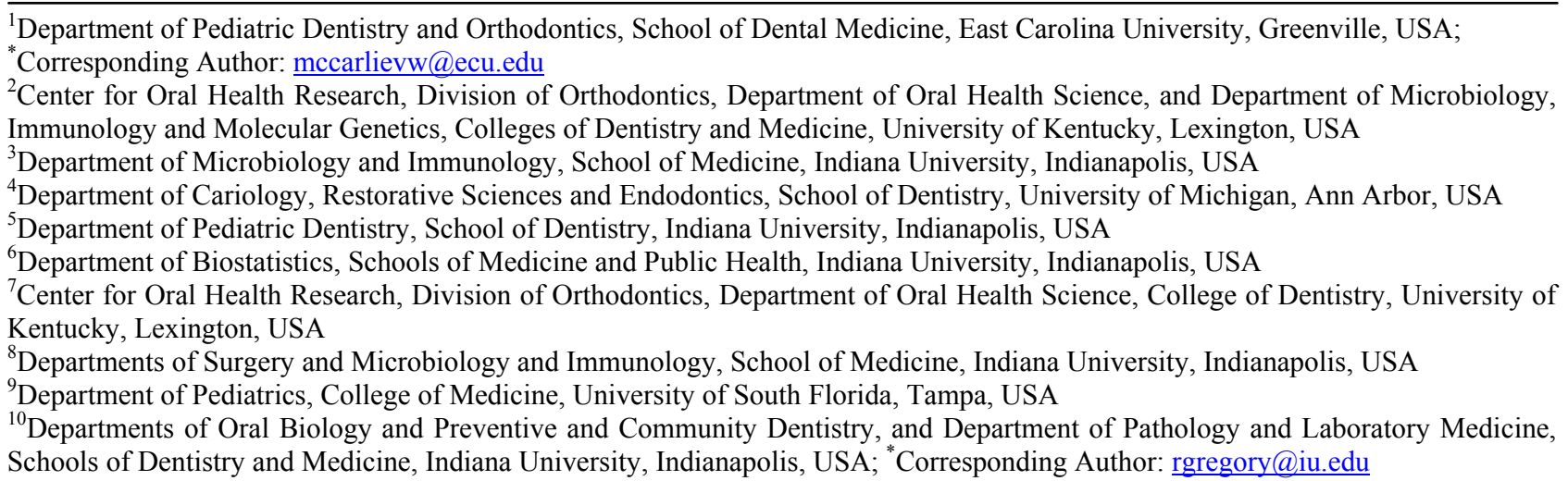

Received 24 May 2013; revised 23 June 2013; accepted 7 July 2013

Copyright (C) 2013 V. Wallace McCarlie et al. This is an open access article distributed under the Creative Commons Attribution License, which permits unrestricted use, distribution, and reproduction in any medium, provided the original work is properly cited.

\section{ABSTRACT}

Bacterial adherence to the acquired dental pellicle, important in dental caries (caries), is mediated by receptor-adhesins such as salivary agglutinin binding to Streptococcus mutans antigen I/II (I/II). Ten selected I/II epitopes were chosen to determine their reactivity to human salivary IgA. Previous studies suggested that a specific HLA biomarker group (HLA-DRB1*04) may have differential influence of immune responses to I/II. However, it was not known whether secretory $\lg A(S \lg A)$ responses to the selected epitopes from HLA-DRB1*04 positive subjects were different compared to controls, or across other caries-related factors such as total $\lg A(\mathrm{Tlg} A)$. Thirty-two total subjects were matched according to HLA type, gender, ethnicity and age. HLA genotyping, oral bacterial, immunoglobulin and antibody analyses were performed. A large observed difference emerged with regard to the natural immune reservoir of TIgA in HLA-DRB1*04 positive subjects, specifically, a $27.6 \%$ reduction compared to controls. In contrast to all other epitopes studied, HLA-DRB1*04 positive sub-

${ }^{\#}$ Dr. Pescovitz, a true mentor, passed away during this work. jects also exhibited reduced reactivity to I/II epitope 834-853. HLA-DRB1*04 positive subjects exhibited lower specific SIgA activity/TIgA to 834-853 and also a lower specific reactivity to 834-853/whole cell $S$. mutans UA159. Furthermore, HLA-DRB1*04 positive subjects exhibited lower responses to I/II in its entirety. The large observed difference in TIgA and the 834-853 reactivity pattern across multiple measures suggest potentially important connections pertaining to the link between HLA-DRB1*04 and caries.

Keywords: Dental Caries; Streptococcus mutans; I/II; IgA; Immunomodulation; Immunogenetics; HLA-II; DRB1; DRB1*04

\section{INTRODUCTION}

The natural history of dental caries (caries) and development of the salivary immune response in humans is multifactorial. Although populated with certain types of oral streptococci (i.e., Streptococcus salivarius and Streptococcus mitis) as newborns, children are not infected with Streptococcus mutans permanently until anywhere from 18 to 36 months [1,2]. Permanent infection with 
mutans streptococci during this period is dominanted by $S$. mutans and S. sobrinus - cariogenic agents in caries. Permanent infection with others of the mutans group does not happen until tooth eruption begins, although we now know this can happen earlier, but colonizing numbers are lower and harder to detect until primary molars start erupting. Newborns lack significant levels of secretory $\operatorname{IgA}(\mathrm{SIgA})$, but by 12 to 24 months most children have SIgA levels similar to those found in adults.

In 2008, a study was published showing that an Iranian cohort of children with a specific human leukocyte antigen (HLA) class II allele (HLA-DRB1*04) were 10 times more likely to have early childhood caries (ECC) [3]. All 79 subjects were between the ages of 12 and 72 months, meaning they would have had time to become permanently infected with mutans streptococci and to have developed a mature level of SIgA. Their individual host antibody responses, however, could have only been as good as their "specificity and functional activity" against the pathogens [4]. The Iranian study did not analyze levels of mutans streptococci colonization or SIgA. However, it did report that of 35 children who did not have ECC, 34 of them were HLA-DRB1*04 negative. As is well known, antibodies, such as SIgA in mucosal immunity, play a major role in immunomodulation and are linked to HLA genetics because intracellular processes and antigen presentation is modulated by particular HLA class II (HLA-II) genes. HLA drives T-cell responses, which stimulates B-cell activation and $\operatorname{IgA}$ secretion. Not only have HLA-II alleles been immunopathogenically associated with members of the streptococcal family [5, 6], but also other infectious bacteria [7].

Animal and human studies suggest that HLA-II genes may play a role in the development of caries. Rat genes corresponding to the HLA region in humans have been found to regulate immunity against $S$. mutans [8]. One report indicated that murine genes found in the $\mathrm{H}-2$ region, corresponding to the HLA region in humans, controlled serum IgG response to particular cariogenic epitopes [9]. The murine $\mathrm{H}-2$ region has a marked effect on clinical caries susceptibility as well [10].

In Caucasian populations, HLA-DRB $1 * 04$ has been suggested as an allele that may increase caries susceptibility $[11,12]$ whereas, in certain Asian and Brazilian populations, HLA-DQB1*06 may be a susceptible allele and HLA-DQB1*02 may be a protective allele in the caries process $[13,14]$. These agree with other reports that identical diseases may be associated with different HLA-II alleles in different populations $[15,16]$.

Animal and human studies suggest that HLA immunogentic interactions are important in modulating a cariogenic infection. Understanding the immunogenetic interactions between host and microbes, such as $S$. mutans, is critical in understanding caries, especially when desir- ing to improve prevention, diagnosis and detection, as well as therapeutic approaches.

Currently, S. mutans "remains prominent in most molecular genetic profiles of incipient dental disease, and thus continues to be a reasonable candidate for elimination" [17]. It also continues to remain the prototypic caries pathogen, and no other species within the oral microbiome "is yet a serious contender to $S$. mutans for a role as a worthwhile marker organism" in the caries process [18]. S. mutans has three surface proteins of major importance, which aid in attaching to tooth surfaces. These include glucosyltransferases (GTFs), glucan-binding proteins (GBPs), and antigen I/II (I/II). These proteins have been vaccine targets for caries. GBPs are surface-associated adhesins that play a substantial role in architectural development of the biofilm, GTFs are cell-associated and secreted enzymes, and I/II is a cell-wall anchored adhesin.

Bacterial adherence to the acquired dental pellicle, important in dental caries, can occur even when other exogenous factors such as sucrose are not present. This is mediated by receptor-adhesins, such as salivary agglutinin, binding to $S$. mutans I/II. Antigen I/II is comprised of several regions (A, V, P and C; Figure 1) that may stimulate salivary IgA reactivity in infected subjects. As mentioned, previous studies suggested that a specific HLA biomarker group (HLA-DRB1*04) may have differential influence on immune responses to I/II. However, it was not known whether secretory IgA (SIgA) responses to the ten selected epitopes from HLA-DRB1*04 positive subjects were different compared to controls. No known published study to date has assessed these questions. The goal of this study was to gain a molecular understanding of $S$. mutans-induced caries with the objective of future vaccine design and biomarker identification

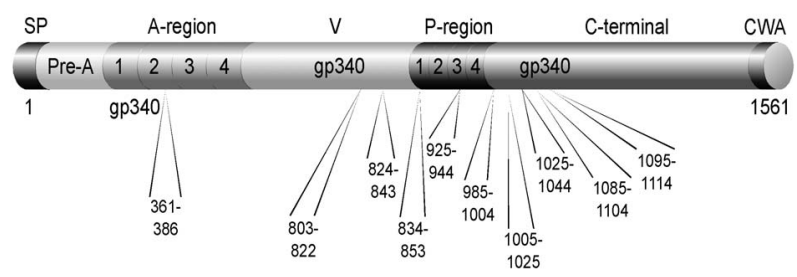

Figure 1. Schematic and diagramatic representations of the surface protein adhesin, antigen I/II. Antigen I/II is comprised of the following regions (and residues): the signal sequence (1-38), a pre-A region (39-120), the A-region (121-447), Vregion (aa 448-839), P-region (840-983), and the C-terminal region (984-1463), and regions for anchor proteins associated with the bacterial cell wall including the LPxTG anchor region (CWA) (1464-1561). The A-region consists of four alanine-rich tandem repeats, specifically A1 (121-201), A2 (202-283), A3 (284-365), and A4 (366-447). The P-region also consists of three to four repeated regions (represented here as four) with about 35 percent proline content, including P1 (840-878), P2 (879-917), P3 (918-956), and P4 (957-983) [26,27]. 
to predict caries susceptibility.

\section{MATERIALS AND METHODS}

\subsection{Bacterial Strains}

S. mutans UA159 (ATCC 700610), NG8 and PC3370 were used in this study. The UA159 genome can be electronically accessed (access \# NC_004350) [19].

\subsection{Study Population}

Prior to this study, all appropriate IRB approvals were secured. Subjects in this study were recruited from the TrialNet Natural History Study (TN NHS), which evaluated healthy yet at-risk for Type I diabetes (T1D) subjects since many of these subjects are HLA-DRB1*04 positive and HLA typing was already done. The study population was divided into two groups: the control group, an HLA-DRB $1 * 04$ negative group $(\mathrm{n}=16)$, and an HLA-DRB $1 * 04$ positive group $(\mathrm{n}=16)$. After completing the informed consent process, subjects were asked to provide a medical history and prescribed demographic information. Subjects who were HLA-DRB1*04 positive (as determined from genotyping during Phase 2 or 3 participation of TN NHS, or as determined by genotyping of genomic DNA from saliva collection as described at the time of the study visit) were invited to participate. Matched gender, ethnicity and age control subjects testing negative for HLA-DRB $1 * 04$ were also invited to participate.

\subsection{Saliva Collection}

At the time of the subject's visit, unstimulated whole saliva was collected and stored at either $-80^{\circ} \mathrm{C}$ (for bacterial enumeration) or $-20^{\circ} \mathrm{C}$ (for antibody assays) until laboratory analysis. In some cases, two samples of saliva were collected (the first for antibody and bacterial analysis and the second for HLA DNA genotyping). For the subjects where DNA had already been collected and HLA sequenced through the TN NHS, and only one saliva sample was required, that sample was collected and later divided in the laboratory into two aliquots, one for bacterial analysis and the other for antibody analysis. Subjects were instructed to provide at least $5 \mathrm{~mL}$ of unstimulated saliva in a $50 \mathrm{~mL}$ sterile tube, and were timed in order to calculate a salivary flow rate. After the samples had been transferred to the laboratory and vortexed, $0.9 \mathrm{~mL}$ was pipetted into another vial and mixed with 0.2 $\mathrm{mL}$ of sterile glycerin, vortexed and stored at $-80^{\circ} \mathrm{C}$ until bacterial analysis. The remainder of the sample was then divided into $1.5 \mathrm{~mL}$ aliquots for antibody analysis and stored at $-20^{\circ} \mathrm{C}$.

For those subjects requiring HLA genotyping (i.e., TN NHS HLA genotyping data was not available), a second sample was collected to determine if the participant was HLA-DRB $1 * 04$ positive or negative. For this purpose, 3 $\mathrm{mL}$ of stimulated saliva (stimulated for $2 \mathrm{~min}$ by chewing on paraffin) was collected with Oragene ${ }^{\circledR}$ DNA sample collection kits (DNA Genotek, Kanata, Ontario, Canada) and was stored at room temperature.

\subsection{HLA Genotyping}

Real time-PCR was utilized to determine group-specific HLA-DRB1 alleles on the Roche ${ }^{\circledR}$ LightCycler $^{\circledR}$ 480 (Roche Diagnostics Corporation, Roche Applied Science, Indianapolis, IN) in the Hereditary Genomics Laboratory at the University of Kentucky. PCR was carried out as previously described [20]. Briefly, each PCR reaction $(10 \mathrm{ul})$ contained: $10 \mathrm{ng}$ of genomic DNA; $1 \mathrm{X}$ of LightCycler480 ${ }^{\circledR}$ FastStart DNA Master Hybridization Probes mix (Roche Diagnostics Corporation); $200 \mathrm{nM}$ HLA-DRB1*04 specific forward primer (5'-GT TTC TTG GAG CAG GTT AAA C-3') tailed with M13f-21 (5'-GTA AAA CGA CGG CCA G-3'); 200 nM DRB-3i reverse primer $\left(5^{\prime}-\mathrm{C}\right.$ TCG CCI CTG CAC IGT IAA GC-3' where I = inosin) tailed with the $\mathrm{M} 13$ reverse sequence (5'-CAG GAA ACA GCT ATG AC-3'); 200 nM HLA-DRB-specific fluorogenic probe

(5'-FAM-labeled-CGG GCG GTG ACG GAG CTG GGG C-Black Hole Quencher-3'); and PCR Boost (Biomatrica, Inc., San Diego, CA). All oligonucleotides were synthesized by Integrated DNA Technologies (IDT; Coralville, Iowa). The RT-PCR cycling consisted of the following steps: a) initial denaturation for 10 minutes at $95^{\circ} \mathrm{C}$; b) 10 cycles of $95^{\circ} \mathrm{C}$ for 5 seconds and $65^{\circ} \mathrm{C}$ for 20 seconds with a single fluorescence reading (465 - 510 $\mathrm{nm})$; c) 25 cycles of $95^{\circ} \mathrm{C}$ for 5 seconds and $60^{\circ} \mathrm{C}$ for 20 seconds with a single fluorescence reading; then d) $40^{\circ} \mathrm{C}$ for 30 seconds cool down. Three cell lines (courtesy of Dr. Janice S. Blum), known to be homozygous for HLADRB1*0401 (Priess human B cell line), heterozygous for HLA-DRB1*04/HLA-DRB1*01 (Frev B cell line), and negative for HLA-DRB1*04 (Sweig, Epstein-Barr virus (EBV) immortalized lymphoblastoid (LCL) B cells; homozygous for HLA-DRB1*1101), were used as controls.

\subsection{Bacterial Analyses}

Aliquots of whole saliva in glycerin were diluted $1: 100$ and $1: 1000$ in sterile saline, double-plated on Mitis Salivarius agar supplemented with sucrose and bacitracin (MSSB) (Anaerobe Systems, Morgan Hill, California). The plates were incubated for 48 hours at $37^{\circ} \mathrm{C}$ and $5 \%$ $\mathrm{CO}_{2}$, and subsequently the number of mutans streptococci colonies enumerated using an automated colony counter (Protocol Synoptic LTD, North Cambridge, United Kingdom), yielding mutans streptococci colony-forming 
units $/ \mathrm{mL}(\mathrm{CFU} / \mathrm{mL})$ of whole saliva.

\subsection{Selecting, Synthesizing, and Storing I/II Peptides}

Ten antigen I/II peptides that induce immune responses in humans, encompassing several regions of the intact protein (Figure 1) were selected for analysis based on immunoreactivity from other studies $[21,22]$. The following peptides were selected:

NAKATYE-AALKQYEADLAAVKKANAA (361-386), IETGKKPNIWYSLNGKIRAV (803-822), VPKVTKEKPTPPVKPTAPTK (824-843), PPVKPTAPTKPTYETEKPLK (834-853), ETEKPLEPAPVEPSYEAEPT (925-944), PTVHFHYFKLAVQPQVNKEI (985-1004), RNNNDINIDRTLVAKQSVVK (1005-1024), FQLKTADLPAGRDETTSFVL (1025-1044), TLATFNADLTKSVATIYPTV (1085-1104), and KSVATIYPTVVGGVLNDGAT (1095-1114). These peptides derived from the A-region (121-447), V-region (448-839), P-region (840-983), and the C-terminal region (984-1463), respectively. Each peptide was synthesized (Peptide 2.0, Chantilly, Virginia) and lyophilized at the following purities (following the same order as above, in percent): 91.1, 92.2, 96.9, 98.0, 95.1, 96.6, 93.6, 80.6, 79.4, and 93.2. Each peptide was dissolved in distilled water to yield a $1 \mathrm{mg} / \mathrm{mL}$ stock solution and stored at $-20^{\circ} \mathrm{C}$.

\subsection{Enzyme-Linked Immunosorbent Assay (ELISA)}

Total IgA, levels of salivary IgA (SIgA) antibodies to I/II epitopes of $S$. mutans UA159, and whole cells of $S$. mutans (UA159, NG8 and I/II-deficient PC3370 on an NG8 background) were detected using ELISA. Briefly, the total level of IgA (TIgA) in saliva was determined by a sandwich ELISA, as follows. Goat anti-human IgA (Sigma Chemical Co., St. Louis, Mo.) was used as the coating antibody and horseradish peroxidase (HRP)labeled goat anti-human IgA (Sigma, alpha chain specific) was used as the detecting antibody as previously described [23]. The plates were read on a SpectraMax 190 microplate spectrophotometer (Molecular Devices, Sunnyvale, California) at $490 \mathrm{~nm}$. IgA standards were used to calculate the unkown IgA sample values by linear regression analysis. Calculations were performed by using the standard curve equation from a $\log$-log function $\left(\mathrm{R}^{2}=\right.$ 0.991). Total IgA determinations were accomplished by creating a standard curve from standard concentrations of human colostral IgA (Sigma: 200 to $0 \mu \mathrm{g} / \mathrm{mL}$ ).

A modified ELISA [24] was used to measure human SIgA binding to the ten I/II peptides $(1 \mu \mathrm{g} / \mathrm{mL})$ and 3 formalin-killed strains of S. mutans (UA159, NG8 and an
NG8-derived antigen I/II deficient mutant strain PC3370). For the latter assays, lysed whole $S$. mutans cells were used in place of I/II epitopes. Data was also reported as a ratio of each peptide value divided by the total IgA concentration or reactivity to the three $S$. mutans whole cell antigens.

\subsection{Data Analysis}

All experiments were done in either duplicate or triplicate and are reported as means and standard errors of the mean. Paired t-tests were used to compare the matched pairs from the HLA-DRB $1 * 04$ positive and control groups. A natural logarithmic transformation of the variables was used for most analyses due to the non-normal distribution of the data.

\section{RESULTS}

Figure 1 represents antigen I/II and the varying locations of the 10 epitopes tested. Previous work has demonstrated that these epitopes are either human salivary IgA targets or elicit varying degrees of human T- and B-cell responses. HLA drives T-cell responses, which stimulates B-cell activation and IgA secretion.

HLA-DRB $1 * 04$ (hereafter, DRB1*04) positive subjects did not exhibit statistically significant differences in levels of $S$. mutans (Figure 2).

Total IgA levels in DRB $1 * 04$ positive subjects were lower by $27.6 \%$ (Figure 3), and although not statistically significant, this observed difference has never been reported. This means that, on average, DRB $1 * 04$ positive subjects have $25 \mu \mathrm{g} / \mathrm{mL}$ less TIgA compared to their negative counterparts. The concentrations of TIgA in all subjects ranged from $15.3-420.4 \mu \mathrm{g} / \mathrm{mL}$, in agreement with a recent report demonstrating TIgA concentrations ranging from $13.7-483.0 \mu \mathrm{g} / \mathrm{mL}$ in 134 healthy individuals [25]. The average control subject exhibited a TIgA concentration of $89 \mu \mathrm{g} / \mathrm{mL}$ whereas the DRB1*04 positive subject had $64 \mu \mathrm{g} / \mathrm{mL}$ of TIgA.

DRB $1 * 04$ positive subjects also exhibited numerically, but not statistically, higher specific immunoreactivity to nine out of 10 epitopes compared to controls (Figure 4). However, DRB $1 * 04$ positive subjects exhibited lessened reactivity to epitope $834-853$, though not statistically significant. This epitope's amino-terminus straddles the carboxy-terminus of the $\mathrm{V}$ region of $\mathrm{I} / \mathrm{II}$, and the epitope's carboxy-terminus encompasses most of the P1 region, which appears to be a very important interface between all three (A, V, and P) I/II regions. Though not statistically significant, DRB $1 * 04$ positive subjects exhibited lower specific SIgA activity/TIgA to 834-853, and also a lower reactivity ratio of 834-853/whole cell $S$. mutans UA159 (Figure 5). In an indirect measure of immunoreactivity against the entire I/II antigen (PC3370 


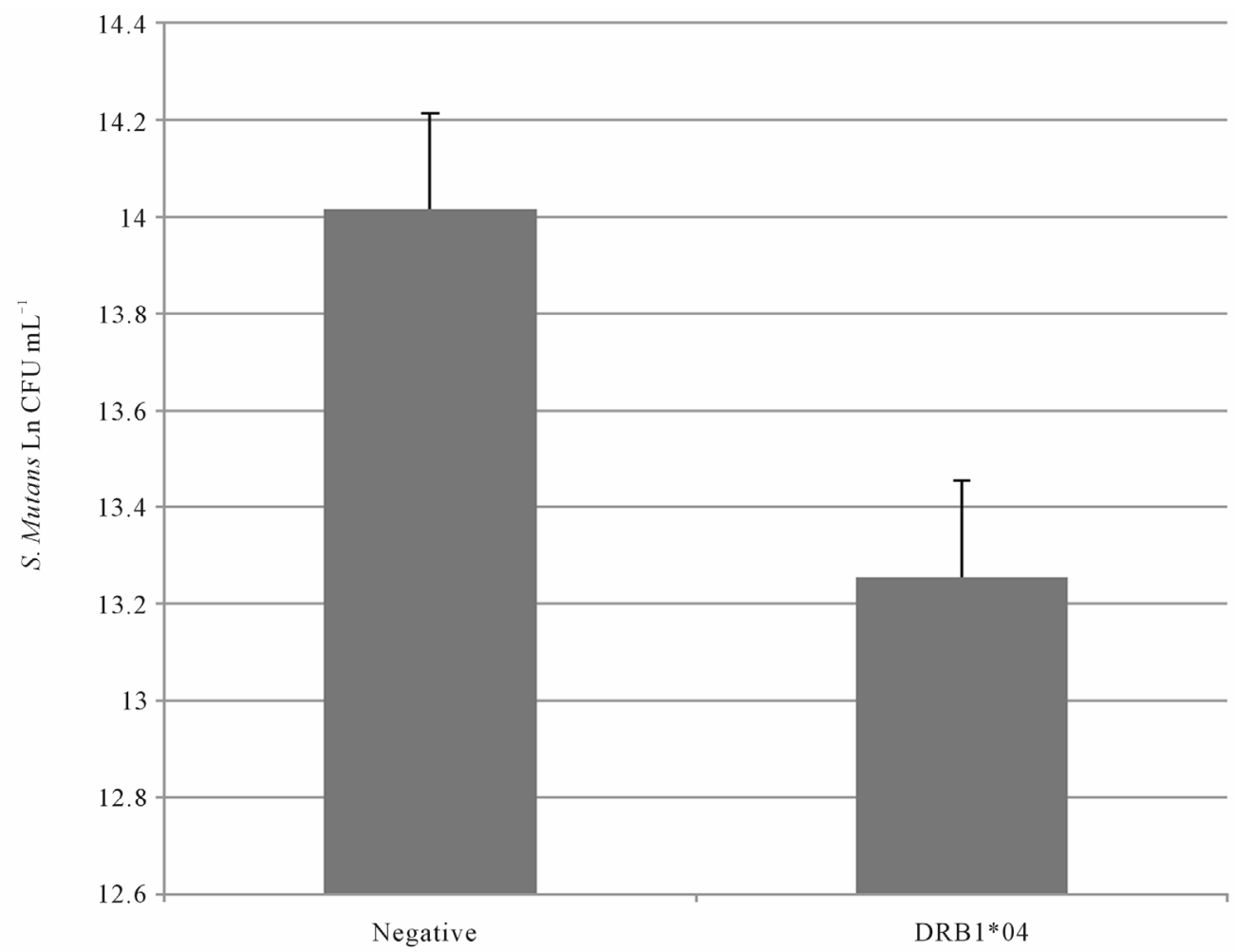

Figure 2. The log transformed number of $S$. mutans colony forming units $/ \mathrm{mL}$ of whole saliva for DRB $1 * 04$ positive $(n=16)$ and negative subjects $(n=16)$. These data are reported as natural $\log$ means and standard errors of the mean.

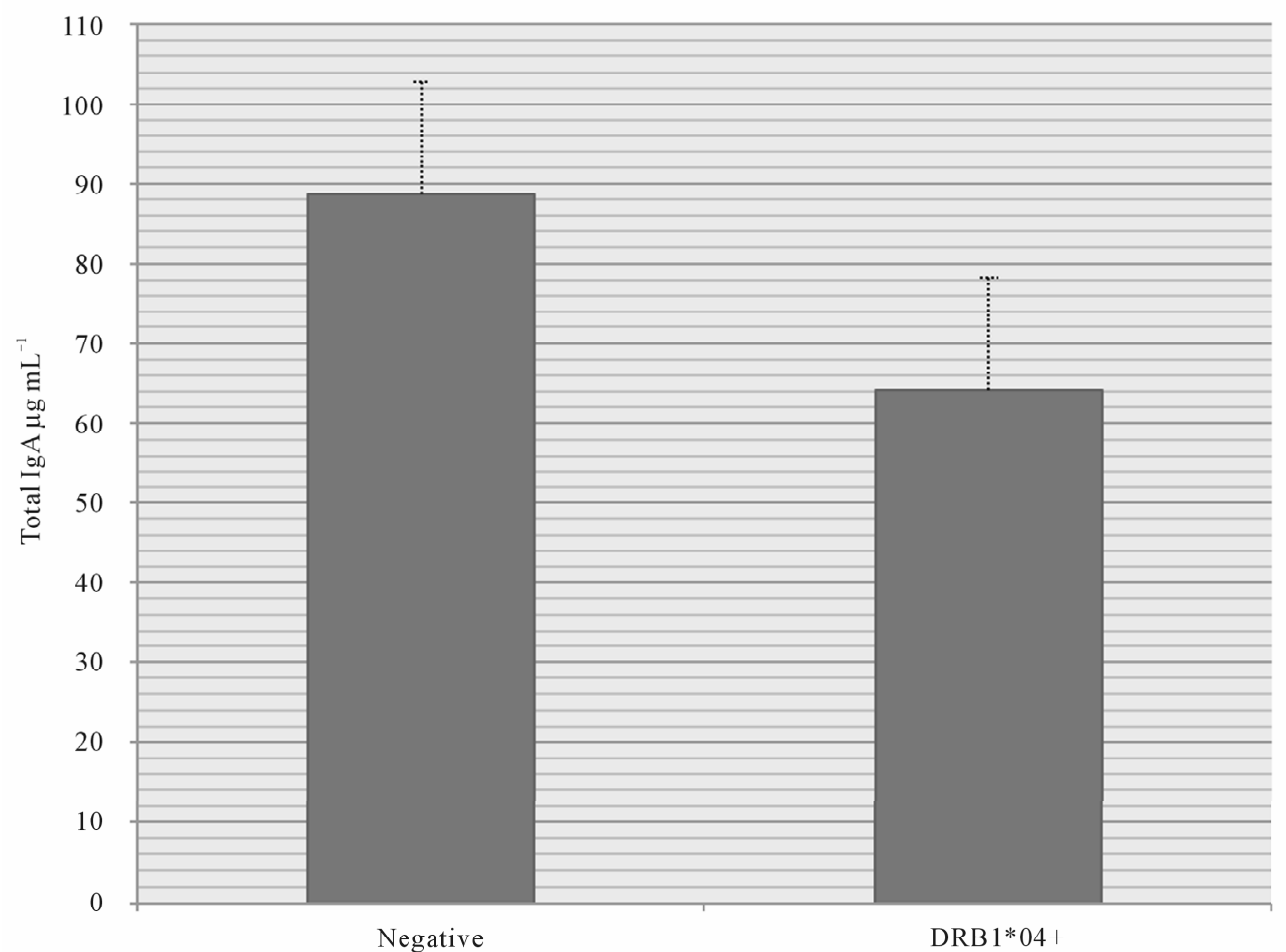

Figure 3. Human salivary total IgA among HLA-DRB1*04 positive $(\mathrm{n}=16)$ and negative subjects $(\mathrm{n}=16)$. 


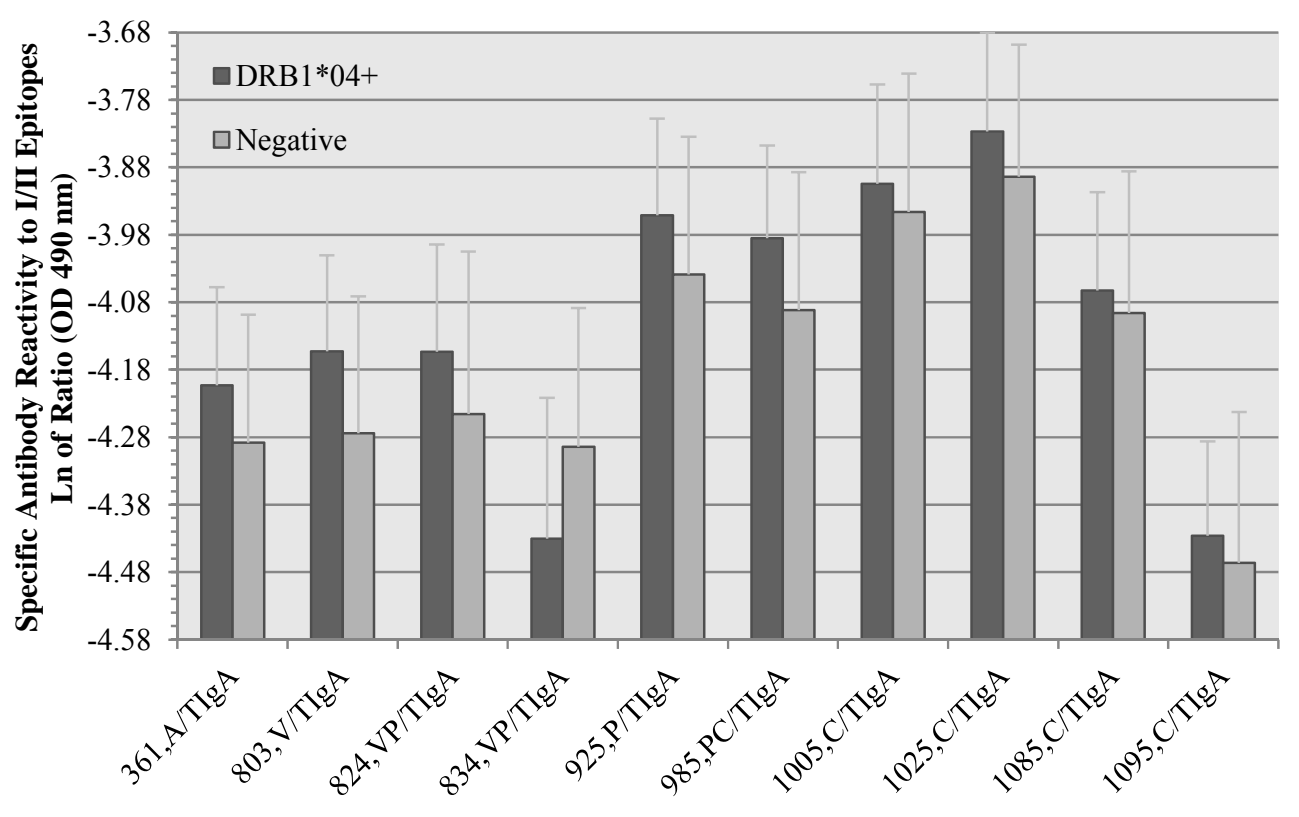

Antigen I/II Epitope

Figure 4. Human salivary IgA specific activity of HLA-DRB $1 * 04$ positive $(\mathrm{n}=16)$ and negative subjects $(\mathrm{n}=16)$ to 10 selected putative epitopes of $S$. mutans I/II. Specific activity was calculated using the natural log of the ratio of each subject's SIgA OD reading for each epitope in triplicate to the total IgA OD for each subject. These data are reported in natural log values as means and standard errors of the mean.

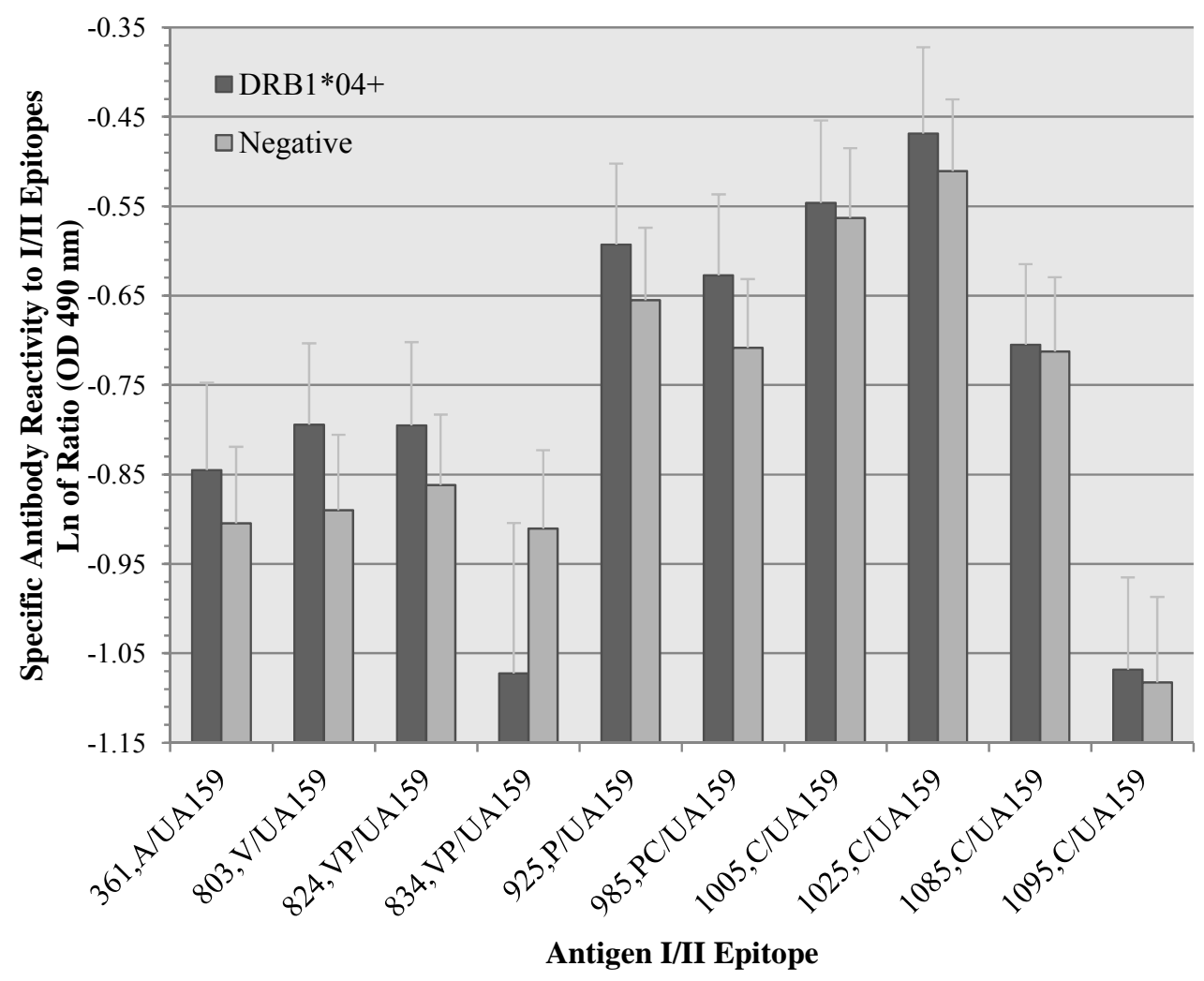

Figure 5. Human salivary IgA reactivity ratios of HLA-DRB $1 * 04$ positive $(\mathrm{n}=16)$ and negative subjects $(\mathrm{n}=16)$ to 10 selected putative epitopes of $S$. mutans $\mathrm{I} / \mathrm{II} / S$. mutans UA159 whole cells (UA159). These data are reported in natural log values as means and standard errors of the mean. 
background devoid of antigen I/II subtracted from the NG8 wildtype strain containing an intact antigen I/II), DRB $1 * 04$ positive subjects exhibited lower responses, though not statistically significant. This suggests that when encountering the entire antigen, in contrast to the selected peptides, these DRB $1 * 04$ positive subjects were not able to respond as well as controls (Figure 6).

\section{DISCUSSION}

The goal of this study was to gain a molecular understanding of $S$. mutans-induced caries with the goal of future vaccine design and biomarker identification to predict caries susceptibility. Previous literature suggested that HLA-DRB $1 * 04$ appears to be a caries susceptibility allele in Caucasians. Our current results do not disprove this notion, but argue for a more nuanced and complicated picture regarding the immunogenetics of caries development.

Consistent with the link between DRB $1 * 04$ and caries, this study revealed a large reduction in TIgA of DRB $1 * 04$ positive subjects $(27.6 \%)$ compared to controls (Figure 3). Though the TIgA difference was not statistically significant, the large observed difference may prove clini- cally important, both to mucosal immunity and caries specifically. Less TIgA putatively leads to a decreased ability to respond to cariogenic organisms.

Also in agreement with the link between DRB1*04 and caries, DRB $1 * 04$ positive subjects exhibited reduced reactivity to a single epitope in this study, 834-853. Though this observed difference was not statistically significant, the fact that this difference occurs across multiple measures suggests that it may be both biologically and clinically important (Figures 4-6). This research needs to be examined in a larger population to verify the differences observed in this study. Future research is necessary to determine if vaccination to this epitope might boost caries immunity since, among other things, 834853 is crucially involved in a primary gp340 binding domain.

Results suggest that DRB1*04 positive subjects exhibit a lower SIgA response to I/II of $S$. mutans NG8 compared to controls (Figure 6). This yet suggests that I/II is important but that the selected epitopes do not account for all immunoreactivity. Both GTFs and GBPs also play an important role in caries development. Researchers have hypothesized that HLA-II alleles produce

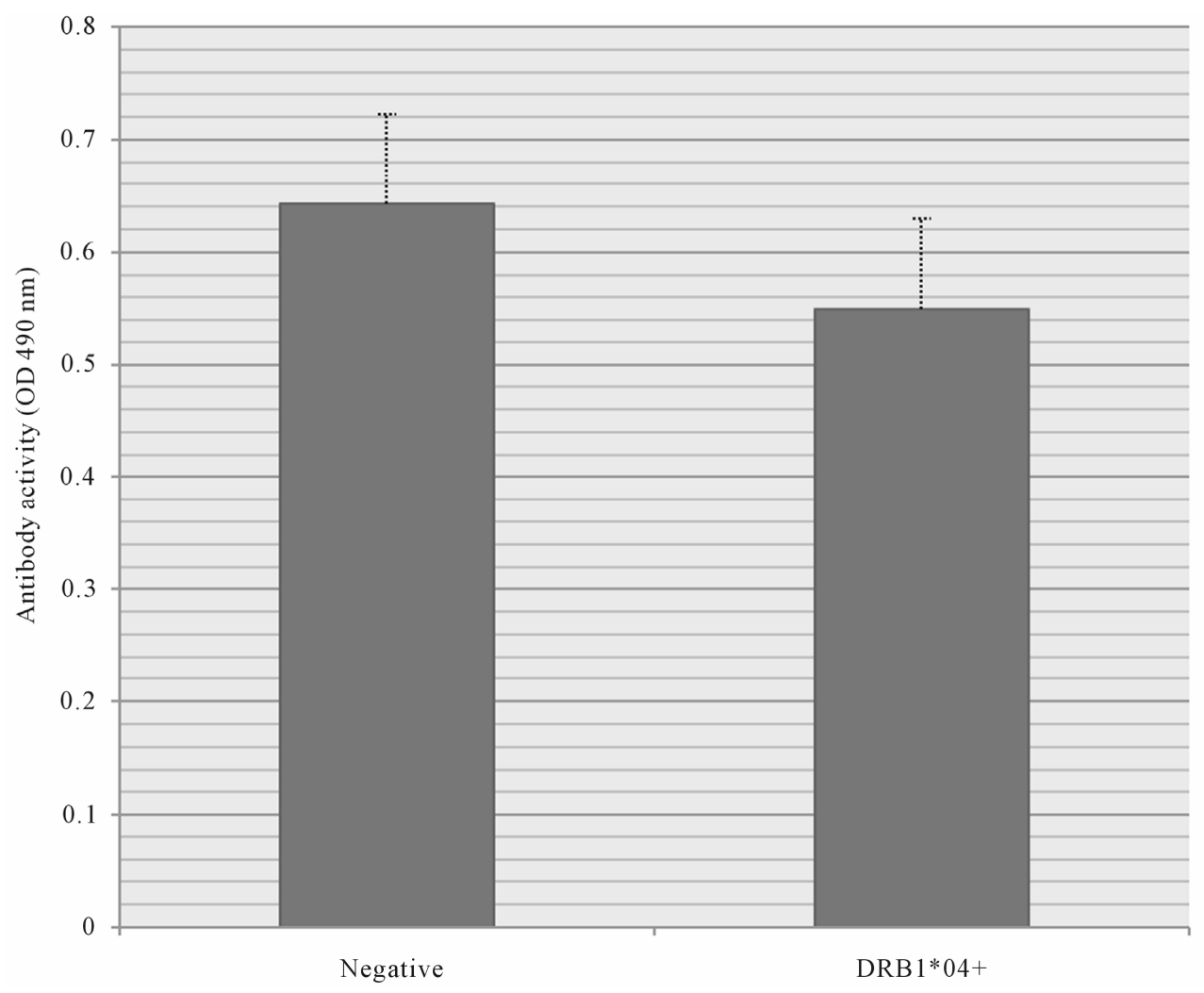

Figure 6. Human salivary IgA immunoreactivity to the entire I/II antigen on NG8 (indirect measure) in HLA-DRB $1 * 04$ positive $(\mathrm{n}=16)$ and negative $(\mathrm{n}=16)$ subjects. Reactivity to NG8 and a I/II-deficient strain (PC3370) was determined and the data were calculated by subtracting OD $490 \mathrm{~nm}$ PC3370 values from NG8 values to focus on epitopes of I/II. These data are reported in optical density absorbance $(490 \mathrm{~nm})$ as means and standard errors of the mean. 
differences in immunological responses to GbpB immunogenic epitopes [28,29]. Nevertheless, results from our lab demonstrate that DRB1*04 positive subjects are slightly higher responders to an I/II-deficient strain (PC 3370 ) of $S$. mutans, as well as to the I/II replete NG8 and UA159 strains (data not shown) than controls. This means that these subjects are immunologically competent to GBPs and other adhesins, compared to controls. Additionally, by subtracting the I/II-deficient strain's reactivity from NG8's activity, an indirect measure of reactivity to $\mathrm{I} / \mathrm{II}$ in its entirety is determined, demonstrating that $\mathrm{I} / \mathrm{II}$ is where $\mathrm{DRB} 1 * 04$ positive subjects may exhibit a lower immune response (Figure 6). This is in agreement with a previous study where two DRB1*04 allelic variants demonstrated lower SIgA reactivity than controls to the entire I/II antigen [12]. Unfortunately, a purified sample of the entire sequence of I/II was not available. This indirect measurement is especially helpful since I/II in its entirety is notoriously unstable and degrades rapidly in vitro; thus it is very difficult to study in isolation as a whole.

It is interesting to note that of all 10 epitopes assayed, one epitope (834-853; which straddles the carboxy-terminus of the $\mathrm{V}$ region and encompasses most of the $\mathrm{P} 1$ region) demonstrated the opposite response across all immunological measures (Figures 4 and 5, and other data not shown) from the other nine epitopes. This epitope was originally identified in a preliminary laboratory study, using pooled saliva, as being most important after normalizing those data (data not shown). These results regarding epitope 834-853 support our initial hypothesis. Taking into account those data suggesting lower reactivity to the entire I/II antigen, 834-853 may be one part of a discontinuous epitope involved in attachment to the acquired salivary pellicle on the tooth surface, and DRB1*04 subjects may have less specificity and functional activity in responding to this epitope.

There is new data that suggests that this may be the case. Although the A and $\mathrm{P}$ regions are important as areas that interact and serve as docking sites for discontinuous epitopes, the variable region is now known to also be very important [26]. Researchers have suggested that I/II presents a lectin-like (i.e., carbohydrate-binding) domain in the middle of the $\mathrm{V}$ region important in bacterial adhesion [30]; for example, in binding fibronectin [31] or "collagen, laminin, keratin, fibrinogen and other oral microorganisms" [32]. It is very interesting to note that 834-853 encompasses the very residues (interacting with 464-471, which was not examined in this study) that make up the distal arm of the $\mathrm{V}$ region (the reactive part of the arm, made up of proximal and distal regions) of what has recently been called a hinge-like area of I/II. This hinge-like area interfaces with all three major regions (A, V, and P) of $\mathrm{I} / \mathrm{II}$ and supports the flexure of what is now known to make up most of the length of the I/II stalk [26]. Not only does the distal arm provide flexing in unison by the $\mathrm{A}, \mathrm{V}$, and $\mathrm{P}$ regions, but it is also involved in governing intermolecular interactions among the backbone of I/II such as solidifying the proximal arm's hydrophobicity, which is locked interiorly by hydrogen bonding and salt-bridges [30]. Even more, the V region exhibits sequence homology with $S$. mutans GbpC, which binds glucan that is synthesized by GTFs and aids development of biofilm. Nevertheless, it is clear that the $\mathrm{V}$ region does not act alone but rather functions in tandem via its distal arm (serving as a sort of center for functionality) with its sister domains.

It is the three-dimensional interaction of the A, V, and $\mathrm{P}$ regions that predominantly affects $S$. mutans attachment to a dental pellicle protein, gp340, alternatively called salivary agglutinin (SAG) or DMBT1 (deleted in malignant brain tumors 1). Human gp340 belongs to a family of innate immune proteins that are characterized by scavenger receptor cysteine-rich (SRCR) proteins. There are SRCR domains along the entire length of gp340, which serve as the binding sites for the specific arrangement of particular sections of the A, V, and P regions within I/II. When in the planktonic form, SRCRs provide gp340 the ability to serve as a pattern recognition receptor [32]. Thus, gp340 "promotes bacterial aggregation and clearance" [33] when it encounters S. mutans planktonically. However, when the glycoprotein is embedded in the tooth pellicle or on the epithelium or on microbes themselves (i.e., immobilized in dental biofilm), it instead serves as a receptor for streptococcal attachment [34].

S. mutans I/II was recently demonstrated to be a singular protein, very different from what has heretofore been understood about other proteins in nature. Researchers demonstrated that it is a hybrid structure of $\alpha$-helices and polyproline type II (PPII) helices intimately associated with each other [26]. PPII helices are more flexible than other regular structures such as $\alpha$-helices or $\beta$-sheets, but are nonetheless solid due to the "rigidity of the proline ring," echoing what was previously said about the functionality of the distal arm of the $\mathrm{V}$ region, which is also linked and a part of the amino terminus of the $\mathrm{P}$ region, whose existence provides a hinge-region allowing the I/II stalk to flex as a unit and yet maintain a $\mathrm{V}$ region hydrophobic core that is locked in place.

What insight does this recently discovered structure provide? For one, it demonstrates that A3 and P1 (and V) interactions, of which $834-853$ is crucially involved, serves as a primary gp340 binding domain. It also demonstrates that a secondary gp340 binding site is located in the C-terminal domain. This means that after the globular $\mathrm{V}$ region (coupled with the amino terminus of 
the $\mathrm{P}$ region and the carboxy terminus of the A region) initially adheres to the salivary pellicle, the distal arm can flex to such a degree that the C-terminal domain, which abuts the anchor motif on $S$. mutans itself, can actually bind gp340 as well. Thus, if host SIgA sufficiently responds to this very particular region, I/II will not be able to take advantage of these binding sites and the process of caries will be abated or slowed. Alternatively, if there were low SIgA responses to this particular epitope located in the distal arm region, it would follow that I/II could take full advantage of its array of virulent mechanisms, which fits the hypothesis with regard to the DRB $1 * 04$ positive group and the epitope found at 834-853. That the control group may exhibit a better ability to naturally fight caries while still in its early stages is supported by the fact of higher reactivity to the 834-853 epitope.

Ultimately, however, how antibodies relate to their HLA gene-encoding counterparts remains to be fully explored. As these fundamental mechanisms become better understood in the long-term [18], our ability to design vaccines will not only increase but also enable us to individually tailor immunizations. Caries is also affected in large part by exogenous variables such as oral hygiene, diet, fluoride exposure, as well as psycho-social factors, all of which may change over time in different ways. If a larger and more comprehensive study confirms what is observed in this study, it will also be necessary to control, to the extent possible, other environmental variables such as those mentioned.

\section{CONCLUSION}

These results show that DRB $1 * 04$ positive subjects have an observed reduction in TIgA compared to controls, an observational difference never before published. Also, it demonstrates that a particular epitope may play a significant role in the caries process depending on the immune status of the host. This epitope (834-853) located in the distal arm of the $\mathrm{V}$ region (also connected to the $\mathrm{P} 1$ region) is probably very important, and provides a biological connection between reduced SIgA reactivity in DRB $1 * 04$ positive subjects to its binding site, and therefore greater adherence to the tooth structure, which can lead to caries.

\section{ACKNOWLEDGEMENTS}

Support in subject accrual and data acquisition for this study was provided by the Type 1 Diabetes TrialNet Study Group at Riley Hospital NIH U01DK085505. Type 1 Diabetes TrialNet Study Group is a clinical trials network funded by the National Institutes of Health (NIH) through the National Institute of Diabetes and Digestive and Kidney Diseases, the National Institute of Allergy and Infectious Diseases, and The Eunice Kennedy Shriver National Institute of Child Health and
Human Development, through the cooperative agreements U01 DK061010, U01 DK061016, U01 DK061034, U01 DK061036, U01 DK061040, U01 DK061041, U01 DK061042, U01 DK061055, U01 DK061058, U01 DK084565, U01 DK085453, U01 DK085461, U01 DK085463, U01 DK085466, U01 DK085499, U01 DK085505, U01 DK085509, and a contract HHSN267200800019C; the National Center for Research Resources, through Clinical Translational Science Awards UL1 RR024131, UL1 RR024139, UL1 RR024153, UL1 RR024975, UL1 RR024982, UL1 RR025744, UL1 RR025761, UL1 RR025780, UL1 RR029890, UL1 RR031986, P30 DK017047, and General Clinical Research Center Award M01 RR00400; the Juvenile Diabetes Research Foundation International (JDRF); and the American Diabetes Association (ADA). The contents of this Article are solely the responsibility of the authors and do not necessarily represent the official views of the NIH, JDRF, or ADA.

This work was supported by an Indiana Clinical and Translational Science Institute (CTSI) award to V.W.M. Some research supplies were also procured with financial support from the Department of Orthodontics and Orofacial Genetics, courtesy of Dr. Kula. The Oral Health Research Institute faculty, staff, and its director, Dr. Zero, provided excellent research infrastructure. Special thanks go to Sue Kelly, Susan Hayden, Sharon Gwinn, Lorena del Carmen Galvez, Jodie Jarrett, Jen Tran, Cunge (Ellen) Zheng and Amir Haider. The two mutant strains, NG8, and an NG8-derived I/II-mutant strain, PC3370, were kindly provided by Dr. L. Jeannine Brady in the Department of Oral Biology, at the University of Florida, Gainesville, Florida.

\section{REFERENCES}

[1] Taubman, M.A. and Nash, D.A. (2006) The scientific and public-health imperative for a vaccine against dental caries. Nature Reviews Immunology, 6, 555-563. doi:10.1038/nri1857

[2] Caufield, P.W., Cutter, G.R. and Dasanayake, A.P. (1993) Initial acquisition of mutans streptococci by infants: Evidence for a discrete window of infectivity. Journal of Dental Research, 72, 37-45. doi:10.1177/00220345930720010501

[3] Bagherian, A., Neamatollahi, H., Afshari, J.T. and Moheghi, N. (2008) Comparison of allele frequency for HLADR and HLA-DQ between patients with ECC and cariesfree children. Journal of Indian Society of Pedodontics and Preventive Dentistry, 26, 18-21. doi:10.4103/0970-4388.40316

[4] Robinette, R.A., Oli, M.W., McArthur, W.P. and Brady L.J. (2011) A therapeutic anti-Streptococcus mutans monoclonal antibody used in human passive protection trials influences the adaptive immune response. Vaccine, 29, 6292-6300. doi:10.1016/j.vaccine.2011.06.027

[5] Boyton, R.J., Smith, J., Jones, M., Reynolds, C., Ozerovitch, L., Chaudhry, A., et al. (2008) Human leucocyte antigen class II association in idiopathic bronchiectasis, a disease of chronic lung infection, implicates a role for adaptive immunity. Clinical \& Experimental Immunology, 152, 95-101. doi:10.1111/j.1365-2249.2008.03596.x

[6] Kotb, M., Norrby-Teglund, A., McGeer, A., El-Sherbini, 
H., Dorak, M.T., Khurshid, A., et al. (2002) An immunogenetic and molecular basis for differences in outcomes of invasive group A streptococcal infections. Nature $\mathrm{Me}$ dicine, 8, 1398-1404. doi:10.1038/nm1202-800

[7] Vanderborght, P.R., Pacheco, A., Moraes, M.E., Antoni, G., Romero, M., Verville, A., et al. (2007) HLA-DRB1* 04 and DRB1*10 are associated with resistance and susceptibility, respectively, in Brazilian and Vietnamese leprosy patients. Genes and Immunity, 8, 320-324. doi:10.1038/sj.gene. 6364390

[8] Niiyama, T., Kojima, H., Mizuno, K., Matsuno, Y., Fujii, H., Misonou, J., et al. (1987) Genetic control of the immune responsiveness to Streptococcus mutans by the major histocompatibility complex of the rat (RT1). Infection and Immunity, 55, 3137-3141.

[9] Takahashi, I., Matsushita, K., Nisizawa, T., Okahashi, N., Russell, M.W., Suzuki, Y., et al. (1992) Genetic control of immune responses in mice to synthetic peptides of a Streptococcus mutans surface protein antigen. Infection and Immunity, 60, 623-629.

[10] Suzuki, N., Kurihara, Y. and Kurihara, Y. (1998) Dental caries susceptibility in mice is closely linked to the H-2 region on chromosome 17. Caries Research, 32, 262-265. doi:10.1159/000016462

[11] Lehner, T., Lamb, J.R., Welsh, K.L. and Batchelor, R.J. (1981) Association between HLA-DR antigens and helper cell activity in the control of dental caries. Nature, 292, 770-772. doi:10.1038/292770a0

[12] Wallengren, M.L.L., Hamberg, K., Ericson, D. and Nordberg, J. (2005) Low salivary IgA activity to cell-surface antigens of mutans streptococci related to HLA-DRB $1 * 04$. Oral Microbiology and Immunology, 20, 73-81. doi:10.1111/j.1399-302X.2004.00192.x

[13] Ozawa, Y., Chiba, J. and Sakamoto, S. (2001) HLA class II alleles and salivary numbers of mutans streptococci and lactobacilli among young adults in Japan. Oral Microbiology and Immunology, 16, 353-357. doi:10.1034/j.1399-302X.2001.160606.x

[14] Valarini, N., Maciel, S.M., Moura, S.K. and Poli-Frederico, R.C. (2012) Association of dental caries with HLA class II allele in Brazilian adolescents. Caries Research, 46, 530-535. doi:10.1159/000341188

[15] Bondinas, G.P., Moustakas, A.K. and Papadopoulos, G.K. (2007) The spectrum of HLA-DQ and HLA-DR alleles, 2006: A listing correlating sequence and structure with function. Immunogenetics, 59, 539-553. doi:10.1007/s00251-007-0224-8

[16] Jiyun, Y., Guisen L., Li Z., Yi S., Jicheng L., et al. (2012) The genetic variants at the HLA-DRB1 gene are associated with primary IgA nephropathy in Han Chinese. BMC Medical Genetics, 59, 539-553.

[17] Smith, D.J. (2010) Dental caries vaccines: Prospects and concerns. Expert Review of Vaccines, 9, 1-3. doi:10.1586/erv.09.143

[18] Russell, R.R.B. (2008) How has genomics altered our view of caries microbiology? Caries Research, 42, 319327. doi: $10.1159 / 000151326$

[19] Ajdić, D., McShan, W.M., McLaughlin, R.E., Savić, G.,
Chang, J., Carson, M.B., et al. (2002) Genome sequence of Streptococcus mutans UA159, a cariogenic dental pathogen. Proceedings of the National Academy of Sciences of the United States of America, 99, 14434-14439. doi:10.1073/pnas.172501299

[20] Danzer, M., Polin, H., Proll, J., Hofer, K., Fae, I., et al. (2007) High-throughput sequence-based typing strategy for HLA-DRB1 based on real-time polymerase chain reaction. Human Immunology, 68, 915-917. doi:10.1016/j.humimm.2007.10.005

[21] Kelly, C.G., Todryk, S., Kendal, H.L., Munro, G.H. and Lehner, T. (1995) T-cell, adhesion, and B-cell epitopes of the cell surface Streptococcus mutans protein antigen I/II. Infection and Immunity, 63, 3649-3658.

[22] Senpuku, H., Tada, A., Nakao, R., Yonezawa, H., Yoneda, S., et al. (2007) Relationships of anti-PAc (361-386) peptide salivary IgA antibody, eosinophils and basophils with periodontal status in the elderly. Fems Immunology and Medical Microbiology, 49, 84-90. doi:10.1111/j.1574-695X.2006.00193.x

[23] Gregory, R.L., Kindle, J.C., Hobbs, L.C., Filler, S.J. and Malmstrom, H.S. (1990) Function of anti-Streptococcus mutans antibodies: Inhibition of virulence factors and enzyme neutralization. Oral Microbiology and Immunology, 5, 181-188. doi:10.1111/j.1399-302X.1990.tb00643.x

[24] Gregory, R.L. (2001) Modified immunogenicity of a mucosally administered antigen. Clinical and Diagnostic Laboratory Immunology, 8, 540-544.

[25] Booth, C.K., Dwyer, D.B., Pacque, P.F. and Ball, M.J. (2009) Measurement of immunoglobulin A in saliva by particle-enhanced nephelometric immunoassay: Sample collection, limits of quantitation, precision, stability and reference range. Annals of Clinical Biochemistry, 46, 401406. doi:10.1258/acb.2009.008248

[26] Larson, M.R., Rajashankar, K.R., Patel, M.H., Robinette, R.A., Crowley, P.J., Michalek, S., et al. (2010) Elongated fibrillar structure of a streptococcal adhesin assembled by the high-affinity association of $\alpha$ - and PPII-helices. Proceedings of the National Academy of Sciences, 107, 59835988. doi:10.1073/pnas.0912293107

[27] Jakubovics, N.S., Kerrigan, S.W., Nobbs, A.H., Stromberg, N, van Dolleweerd, C.J., Cox D.M., et al. (2005) Functions of cell surface-anchored antigen I/II family and Hsa polypeptides in interactions of Streptococcus gordonii with host receptors. Infection and Immunity, 73, 6629-6638. doi:10.1128/IAI.73.10.6629-6638.2005

[28] Nogueira, R.D., Alves, A.C., Napimoga, M.H., Smith D.J. and Mattos-Graner, R.O. (2005) Characterization of Salivary immunoglobulin A responses in children heavily exposed to the oral bacterium Streptococcus mutans: Influence of specific antigen recognition in infection. Infection and Immunity, 73, 5675-5684. doi:10.1128/IAI.73.9.5675-5684.2005

[29] Smith, D.J., King, W.F., Barnes, L.A., Peacock, Z. and Taubman, M.A. (2003) Immunogenicity and protective immunity induced by synthetic peptides associated with putative immunodominant regions of Streptococcus mutans glucan-binding protein B. Infection and Immunity, 71, 1179-1184. doi:10.1128/IAI.71.3.1179-1184.2003 
[30] Troffer-Charlier, N., Ogier, J., Moras, D. and Cavarelli, J. (2002) Crystal structure of the V-region of Streptococcus mutans antigen I/II at 2.4 angstrom resolution suggests a sugar preformed binding site. Journal of Molecular Biology, 318, 179-188. doi:10.1016/S0022-2836(02)00025-6

[31] Jakubovics, N.S., Brittan, J.L., Dutton, L.C. and Jenkinson, H.F. (2009) Multiple adhesin proteins on the cell surface of Streptococcus gordonii are involved in adhesion to human fibronectin. Microbiology, 155, 3572-3580. doi:10.1099/mic.0.032078-0

[32] McArthur, W.P., Rhodin, N.R., Seifert, T.B., Oli, M.W., Robinette, R.A., Demuth, D.R., et al. (2007) Characterization of epitopes recognized by anti-Streptococcus mu- tans P1 monoclonal antibodies. Fems Immunology and Medical Microbiology, 50, 342-353.

doi:10.1099/mic.0.032078-0

[33] Loimaranta, V., Hytonen, J., Pulliainen, A.T., Sharma, A., Tenovuo, J., Stromberg, N., et al. (2009) Leucine-rich repeats of bacterial surface proteins serve as common pattern recognition motifs of human scavenger receptor gp340. Journal of Biological Chemistry, 284, 1861418623. doi:10.1074/jbc.M900581200

[34] Nobbs, A.H., Lamont, R.J. and Jenkinson, H.F. (2009) Streptococcus adherence and colonization. Microbiology and Molecular Biology Reviews, 73, 407-450. doi:10.1128/MMBR.00014-09 